\title{
L'écriture féminine à la Renaissance française sous le regard des chercheurs canadiens
}

\author{
DIANE DESROSIERS \\ Université McGill \\ JEAN-PHILIPPE BEAULIEU \\ Université de Montréal
}

\begin{abstract}
Au cours des dernières décennies, un travail considérable a été accompli par les chercheurs canadiens dans l'exhumation et la réhabilitation des textes des femmes de lettres de la Renaissance française. En raison de leur positionnement géographique à l'intersection des États-Unis et de l'Europe, ces chercheurs participent à la fois des avancées de la recherche française en matière d'érudition et des percées théoriques de la réflexion nord-américaine, enrichie par les gender studies et les études culturelles. Cela explique en partie le caractère original de leur contribution à ce champ de recherche, tant par le choix de leur objet d'étude et l'exploration de corpus marginaux que par l'approche critique et le positionnement théorique qu'ils ont adoptés. Le présent article vise à cerner la spécificité des activités de recherche menées en langue française au Canada dans les domaines bibliographique, éditorial et critique, en portant une attention particulière aux enjeux théoriques et idéologiques des choix effectués par les chercheurs.
\end{abstract}

Over the past few decades, significant work has been accomplished by Canadian researchers unearthing and recovering texts by the women writers of the French Renaissance. Because of their geographical location-at the intersection of the United States and Europe-these researchers share simultaneously in the advances of French research in the discipline of scholarship and in the theoretical breakthroughs of North American thought informed by gender and cultural studies. This explains, in part, the unique character of their contribution to this field of research - as much for their chosen object of study and exploration of a marginal corpus of work as by their adoption of a certain critical approach and theoretical position. This article seeks to outline the specific features of research activities undertaken in the French language in Canada in the areas of bibliography, editing, and criticism, highlighting in particular the theoretical and ideological issues at stake in the choices made by the researchers.

Pour bien prendre la mesure des transformations radicales intervenues
dans le champ des études portant sur l'écriture des femmes aux XVI et XVII e siècles français, rappelons qu'il y a à peine vingt ans, un éditeur européen refusait de publier les écrits d'Hélisenne de Crenne en invoquant la lourdeur de 
son style qu'il comparait à celui de l'écolier limousin ${ }^{1}$. Et il était encore courant, au début des années 1990, de trouver des propos condescendants soulignant la piètre valeur des œuvres de Marie de Gournay dans lesquelles on ne voyait qu'une pâle reflet des Essais de Montaigne. Cependant, un travail considérable a été accompli au cours des deux dernières décennies, notamment par les seiziémistes canadiens qui ont joué un rôle important dans l'exhumation et la réhabilitation des textes signés par des femmes de lettres de la Renaissance française. Leurs contributions se caractérisent tant par le choix de leur objet d'étude et l'exploration de corpus considérés marginaux (par exemple, la correspondance et les écrits religieux) que par l'approche critique et le positionnement théorique que ces chercheurs adoptent. En effet, en raison de leur situation géographique, au carrefour des États-Unis et de l'Europe, ils participent à la fois des avancées de la recherche française en matière d'érudition historique et des percées théoriques de la réflexion nord-américaine enrichie par les gender studies et les études culturelles. Sous cet éclairage, nous nous proposons de cerner la spécificité des activités de recherche menées au Canada, ce qui suppose une réflexion - forcément succincte dans les limites de cet état des lieux - sur les enjeux théoriques et idéologiques des choix effectués par les spécialistes.

Bien que les seiziémistes canadiens qui s'intéressent à la littérature française de la Renaissance soient relativement peu nombreux, une proportion non négligeable d'entre eux s'est penchée, à un moment ou à un autre, sur les écrits féminins, donnant à ce secteur d'étude un essor qui n'a pas été sans retombées chez nos voisins du Sud, comme chez les chercheurs européens, plus spécifiquement ceux de l'Hexagone. En effet, à une époque où travailler sur ce corpus textuel était considéré comme une entreprise de piètre intérêt, quelques Canadiennes se sont aventurées sur ce terrain, en dépit des résistances institutionnelles. On pense, par exemple, à Hannah Fournier dont la thèse de doctorat proposait une analyse stylistique des poèmes de Marguerite de

1. Ces préventions expliquent probablement que la thèse de doctorat d'Harry R. Secor, professeur à l'Université de Toronto, n'ait jamais été publiée. Elle consistait en une édition critique des Angoysses douloureuses d'Hélisenne de Crenne, dont tout l'apparat critique était rédigé en français : Harry $\mathrm{R}$. Secor, Helisenne de Crenne: Les Angoysses douloureuses qui procedent d'Amours (1538): A Critical Edition based on the Original Text with Introduction, Notes and Glossary (New Haven : Yale University, 1957). 
Navarre $^{2}$. Par le seul choix de ce corpus et sans nécessairement se réclamer d'une lecture féministe, cette étude constituait, dès la fin des années 1960, une affirmation de la valeur et de la validité d'un tel ensemble textuel. Dans le domaine des écrits attribués à des femmes au début de la première modernité, nous n'avons repéré que quatre mémoires de maîtrise produits dans les universités canadiennes au cours des années $1970^{3}$ et une thèse de doctorat, celle de Jean-Philippe Beaulieu, soutenue en $1988^{4}$. Il faut attendre le début de la décennie suivante pour voir se multiplier des mémoires et des thèses ayant trait aux écrits féminins ${ }^{5}$, en partie sous l'influence de quelques pionnières : Jane Couchman au Collège Glendon de l'Université York, Hannah Fournier à l'Université de Waterloo, Madeleine Jeay à l'Université McMaster, Eva Kushner à l'Université McGill' ${ }^{6}$ puis au Collège Victoria (Université de Toronto), Elaine Limbrick à l'Université de Victoria et Simone Maser à l'Université d'Ottawa. À divers degrés, ces professeures ont préparé le terrain, en légitimant, par leur enseignement, leur recherche et la direction de mémoires de maîtrise et de thèses de doctorat, des objets d'étude considérés jusque-là comme tout à fait secondaires. Travaillant de façon souvent isolée, elles ont formé plusieurs générations d'étudiants. La publication du collectif Paroles rebelles qui réunissait des contributions de Jane Couchman, Nicole Mallet, Marie-France

2. Hannah Vickers Fournier, Style in the Non-Dramatic Verse of the Marguerites de la Marguerite des Princesses (London : University of Western Ontario, 1976).

3. Jean Bowman, La Comedie sur le trepas du Roy de Marguerite de Navarre (Winnipeg : University of Manitoba, 1974) ; Lise Trudel, Les idées féministes de Christine de Pizan (Montréal : Université McGill, 1974) ; Lisette Girouard, Étude des Comptes amoureux de Jeanne Flore (Montréal : Université McGill, 1977) ; et Paula J. O. Read, L'allégorie dans la Mutation de Fortune de Christine de Pisan (Fredericton : University of New Brunswick, 1979).

4. Jean-Philippe Beaulieu, Le didactisme amoureux et sa réalisation textuelle dans les œuvres d'Hélisenne de Crenne (Ottawa : Université d'Ottawa, 1988).

5. Signalons, entre autres, au tout début des années 1990, la thèse de doctorat de Katy Emck, " $A$ Wanton Woman and a Wise:" Women Writing about Desire in Renaissance Europe, 1540-1620 (Edmonton: University of Alberta, 1994) et celle de Mary Ann Addy Maxwell, "Speak that I may see you:" Concealment and Unconcealment in the Colloquia of Erasmus, Marguerite de Navarre's Heptaméron and Sydney's New Arcadia (Toronto: University of Toronto, 1993). Après 1995, on voit se multiplier les thèses portant plus spécifiquement sur des corpus de langue française.

6. Mentionnons également la contribution de Giuseppe Di Stefano et d'Isida Cremona au Département de langue et littérature françaises de l’Université McGill. 
Silver et Christine Klein-Lataud a marqué en 1992 un moment important de cette reconnaissance des corpus féminins ${ }^{7}$.

Progressivement s'est donc fait sentir un effet fédérateur s'exprimant dans l'établissement de collaborations et de réseaux qui se sont concrétisés avec la création du groupe de recherche MARGOT ${ }^{8}$ et la tenue du colloque organisé en 1993 à Waterloo par Hannah Fournier et Jean-Philippe Beaulieu'. Si l'on exclut les rares colloques portant spécifiquement sur des femmes de lettres notoires (Marguerite de Navarre ou Louise Labé), le colloque de Waterloo a été le premier, sur la scène internationale, à avoir pour unique objet les femmes écrivains d'Ancien Régime ${ }^{10}$. Ce colloque a donné le coup d'envoi à toute une série de rencontres qui se sont d'abord déroulées en Amérique du Nord (Saint Louis [Missouri], 1995 ; Montréal, 1997 ; Charlottesville [Virginie], 1999) ${ }^{11}$, avant que des chercheurs européens ne prennent la relève (Rennes, 2002 ; Rouen, 2005) ${ }^{12}$. C'est à l'occasion des échanges survenus lors du colloque de Montréal en 1997 qu'a été exprimé le souhait que soit mise sur pied une association permettant de centraliser

7. Marguerite Andersen et Christine Klein-Lataud, éd., Paroles rebelles (Montréal : Les Éditions du remue-ménage, 1992).

8. Voir le site de ce groupe de recherche, qui est toujours actif, à l'adresse électronique suivante : https:// uwaterloo.ca/margot/ Le quatrième colloque international MARGOT s'est déroulé au Bernard College, à New York, du 18 au 20 juin 2014, sous le thème "Women and Community in the Ancien Régime: Traditional and New Media ».

9. Le collectif résultant de ce colloque a été publié par Atlantis, la revue canadienne d'études féministes : Jean-Philippe Beaulieu et Hannah Fournier, éd., Femmes et textes sous l'Ancien Régime / Women and Texts in Pre-Revolutionary France, Atlantis 19.1 (1993).

10. Bien qu'accueillant occasionnellement des communications consacrées aux écrits féminins d'Ancien Régime, les rencontres annuelles tenues entre 1984 et 1996 sous la désignation " Continental LatinAmerican and Francophone Women Writers » à la Wichita State University (Kansas) portaient sur des corpus plus larges.

11. Colette H. Winn et Donna Kuizenga, éd., Women Writers in Pre-Revolutionary France: Strategies of Emancipation (New York: Garland, 1997) ; Jean-Philippe Beaulieu et Diane Desrosiers-Bonin, éd., Dans les miroirs de l'écriture. La réflexivité chez les femmes écrivains d'Ancien Régime (Montréal : Université de Montréal, Département d'études françaises, coll. Paragraphe, 1998). Le colloque de Charlottesville organisé par Mary McKinley en 1999 avait pour thème Les femmes et la culture en transition / Women and Cultural Change.

12. Isabelle Brouard-Arends, éd., Lectrices d'Ancien Régime (Rennes : Presses universitaires de Rennes, 2003) ; Sylvie Steinberg et Jean-Claude Arnould, éd., Les femmes et l'écriture de l'histoire, 1400-1800 (Mont-Saint-Aignan : Presses universitaires de Rouen et du Havre, 2008). 
les renseignements relatifs aux activités des chercheurs et servant de vitrine à ce secteur en émergence. Ce sont les efforts d'Éliane Viennot qui ont, quelques années plus tard, donné forme à ce regroupement de chercheurs sous l'acronyme SIEFAR ${ }^{13}$, devenu depuis la pierre angulaire des études sur les femmes d'Ancien Régime, l'équivalent, pour l'aire culturelle française, de la Society for the Study of Early Modern Women ${ }^{14}$. Plusieurs rencontres ont eu lieu par la suite en terre canadienne : le colloque Dix ans de recherche sur les femmes écrivains de l'Ancien Régime organisé à l'Université de Waterloo en 2005 sous la présidence d'honneur d'Hannah Fournier ${ }^{15}$, le colloque du Centre for Reformation and Renaissance Studies de l'Université de Toronto tenu la même année autour du thème Creating Women : Notions of Femininity from 1350 to $1700^{16}$ et celui de l'Université du Québec à Rimouski en 2007 : Femmes, rhétorique et éloquence sous l'Ancien Régime ${ }^{17}$. Quatre journées d'études portant sur la Querelle des femmes ont pris le relais entre 2008 et 2011, cette fois à Paris et à New York ${ }^{18}$.

13. La Société Internationale pour l'Étude des Femmes de l'Ancien Régime (SIEFAR) a été créée à Paris en 2000. Voir son site à l'adresse électronique suivante : http://www.siefar.org/

14. Linda Woodbridge, auteure du classique Women and the English Renaissance: Literature and the Nature of Womankind (1540-1620) (Urbana : University of Illinois Press, 1984) et alors professeure à l'Université de l'Alberta, faisait partie du Comité organisateur de la Society for the Study of Early Modern Women, fondée en 1993 aux États-Unis. Jane Couchman, professeure émérite du Collège Glendon, est la présidente sortante de cette association. Voir le site électronique de la SSEMW : http:// ssemw.org/

15. Guy Poirier, Christine McWebb, François Paré et Delbert W. Russel, éd., Dix ans de recherche sur les femmes écrivains de l'Ancien Régime : influences et confluences. Mélanges offerts à Hannah Fournier (Québec : Presses de l'Université Laval, 2009).

16. Manuela Scarci, éd., Creating Women. Representation, Self-Representation and Agency in the Renaissance (Toronto : Centre for Reformation and Renaissance Studies, 2013).

17. Claude La Charité et Roxanne Roy, éd., Femmes, rhétorique et éloquence sous l'Ancien Régime (SaintÉtienne : Publications de l'Université de Saint-Étienne, 2012).

18. On pourra consulter les quatre volumes déjà parus de la série « Revisiter la Querelle des femmes » (Saint-Étienne : Publications de l’Université de Saint-Étienne) : Éliane Viennot et Nicole Pellegrin, éd., Discours sur l'égalité / inégalité des femmes et des hommes, de 1750 aux lendemains de la Révolution (2012); Danielle Haase-Dubosc et Marie-Elisabeth Henneau, éd., Discours sur l'égalité / inégalité des sexes, de 1600 à 1750 (2013) ; Armel Dubois-Nayt et Nicole Dufournaud, éd., Discours sur l'égalité / inégalité des sexes, de 1400 à 1600 (2013) ; Florence Rochefort et Éliane Viennot, éd., L'engagement des hommes pour l'égalité des sexes, XIVe-XXI siècles (2013). 
À travers cette floraison d'échanges, on peut percevoir l'impulsion communiquée au domaine par les chercheurs canadiens qui, au cours des années 1990, ont éprouvé avec une sensibilité particulière la nécessité d'explorer ce qui était encore des terrae incognitae pour reprendre le titre d'un collectif préparé par les soins de Marie-Laure Girou Swiderski (Université d’Ottawa) ${ }^{19}$. Cette exploration s'est effectuée sur trois fronts : bibliographique, éditorial et analytique. Il a d'abord été nécessaire d'identifier et de localiser la production scripturaire des femmes d'expression française sous l'Ancien Régime. On a donc répertorié les textes imprimés, signés par des femmes ou attribués à des figures féminines, étape indispensable à toute entreprise critique. L'équipe composée entre autres de Pierre-Louis Vaillancourt et Marie-Laure Girou Swiderski a réalisé un travail pionnier dont l'un des fruits a été l'établissement du répertoire des écrits féminins non fictionnels antérieurs à la Révolution française ${ }^{20}$. Soulignons aussi le dépouillement bibliographique exhaustif entrepris par William Kemp qui a inventorié les incunables et les imprimés portant la signature de femmes publiés avant $1574^{21}$.

Parallèlement à ces activités bibliographiques, il a également fallu rendre accessibles certains de ces ouvrages qui, pour la plupart, n'avaient pas connu de réédition depuis leur publication initiale. Les chercheurs canadiens ont donc produit de nombreuses éditions critiques et procuré des adaptations de ces textes en version moderne. Jean-Philippe Beaulieu a contribué de manière substantielle à ce travail éditorial. En collaboration avec Hannah Fournier, il a publié l'édition critique des Epistres familières et invectives d'Hélisenne de

19. Marie-Laure Girou Swiderski, éd., Terrae incognitae de l'écriture féminine (Toronto : Legas, 2004).

20. Marie-Laure Girou Swiderski, Claire Le Brun-Gouanvic, Pierre-Louis Vaillancourt et Marie-France Wagner, 10 décembre 2005, Les écrits féminins non fictionnels du Moyen âge au XVII siècle : un inventaire raisonné, http://aix1.uottawa.ca/ margirou/index.htm ; Pierre-Louis Vaillancourt et Dawn Smyth, Les écrits féminins non fictionnels de la Renaissance. Un inventaire descriptif (Ottawa : Legas, 2003); PierreLouis Vaillancourt et Marie-Laure Girou Swiderski, La production littéraire féminine d'Ancien Régime. Répertoire bibliographique (Ottawa : Legas, 1997).

21. Une première tranche de cette bibliographie concernant les écrits de femmes publiés jusqu'en 1549 a paru dans un numéro désormais épuisé de la revue Littératures: William Kemp, "Textes composés ou traduits par des femmes et imprimés en France avant 1550 ", in L'Écriture des femmes à la Renaissance française, éd. Diane Desrosiers-Bonin, Littératures 18 (1998) : 151-220. La deuxième partie de ce travail bibliographique couvre la période de 1550 à 1574 : William Kemp, Hélène Hotton et Christian Veilleux, «Bibliographie des imprimés féminins de 1550 à 1574 ", in L'Écriture des femmes à la Renaissance française 2, éd. Diane Desrosiers et Christian Veilleux, Littératures 28 (2014) : 101-239. 
Crenne et, avec Diane Desrosiers, celle du Songe de Madame Helisenne. Il a aussi réalisé la version modernisée du triptyque que forment ces deux textes et Les Angoysses douloureuses qui procedent d'amours ${ }^{22}$. De concert avec Hannah Fournier à qui l'on doit également une édition des Jumeaux martyrs de Madame de Saint-Balmon ${ }^{23}$, il a établi le texte des Advis de Marie de Gournay ${ }^{24}$. Cette publication témoigne d'un intérêt nouveau pour la fille d'alliance de Montaigne, dont Constant Venesoen, professeur à la University of Western Ontario, a édité l'essai sur l'Égalité des hommes et des femmes, le Grief des dames et le Proumenoir de monsieur de Montaigne de même qu'un ensemble de discours sur la médisance ${ }^{25}$. Sous le titre Remontrances, prophéties et confessions de femmes (1575-1650), Jean-Philippe Beaulieu a récemment constitué un florilège de textes au nombre desquels figurent des pièces portant notamment les noms d'Anne d'Este, Catherine de Clèves, Marguerite d'Auge et Léonora Galigai ${ }^{26}$. De son côté, Claire Le Brun a fait paraître le Chemin de long estude de Dame Cristine de Pise édité par Jean Chaperon en $1549^{27}$. L'édition critique des

22. Jean-Philippe Beaulieu et Hannah Fournier, éd., Les Epistres familières et invectives de ma dame Hélisenne (Montréal : Les Presses de l’Université de Montréal, 1995) ; Jean-Philippe Beaulieu et Diane Desrosiers-Bonin, éd., Le Songe de Madame Helisenne (Paris : Champion, 2006) ; Jean-Philippe Beaulieu, éd., Les Épitres familières et invectives; Le Songe (Saint-Étienne : Publications de l'Université de Saint-Étienne, 2008) ; Jean-Philippe Beaulieu, éd., Les Angoisses amoureuses qui procèdent d'amour (Saint-Étienne : Publications de l’Université de Saint-Étienne, 2005).

23. Carmeta Abbott et Hannah Fournier, éd., Madame de Saint-Balmon, Les Jumeaux martyrs (Genève : Droz, 1995).

24. Jean-Philippe Beaulieu et Hannah Fournier, éd., Marie le Jars de Gournay, Les Advis, ou, les Presens de la demoiselle de Gournay (Amsterdam : Rodopi, vol. I, 1997 ; vol. II, 2002).

25. Constant Venesoen, éd., Marie de Gournay, Égalité des hommes et des femmes ; Grief des dames, suivis du Proumenoir de monsieur de Montaigne (Genève : Droz, 1993) ; Constant Venesoen, éd., Marie de Gournay, Textes relatifs à la calomnie (Tübingen : G. Narr Verlag, 1998). Voir aussi du même critique ses Études sur la littérature féminine du XVII siècle : Mademoiselle de Gournay, Mademoiselle de Scudéry, Madame de Villedieu, Madame de Lafayette (Birmingham : Summa, 1990) et ses plus récents ouvrages : Madame de Maintenon, sans retouches (Tübingen : G. Narr Verlag, 2012) et Les Loisirs de Madame de Maintenon : études et textes (Paris : Classiques Garnier, 2011). Il est aussi l'éditeur de plusieurs textes de Madame de Pringy (Paris : Champion, 2002), de Madame de Brégy (Paris : Champion, 2006) et de la correspondance d'Anne Marie de Schurman (Paris : Champion, 2004).

26. Jean-Philippe Beaulieu avec la collaboration de Diane Desrosiers et Claude La Charité, Remontrances, prophéties et confessions de femmes (1575-1650) (Paris : Classiques Garnier, 2014).

27. Claire Le Brun, éd., Jean Chaperon, Le Chemin de long estude de Dame Cristine de Pise [1549] (Paris : Champion, 2008). 
œuvres de Marie de Romieu que Claude La Charité a amorcée dans sa thèse de doctorat $^{28}$ est en voie de complétion. La participation des femmes à la Réforme évangélique puis aux mouvements protestants de dissidence religieuse a aussi suscité son lot d'éditions. Le Groupe d'analyse et de recherche sur l'écriture des femmes au XVI ${ }^{\text {e }}$ siècle (GARSE-XVI) devrait livrer prochainement l'édition annotée de l'épître de Marie d'Ennetières à Marguerite de Navarre ${ }^{29}$. Quant à Eugénie Pascal, dont la thèse de doctorat explorait la correspondance des princesses tant catholiques que protestantes à la fin du $\mathrm{XVI}^{\mathrm{e}}$ siècle et au début du XVII ${ }^{\mathrm{e}}$ siècle, elle prépare maintenant l'édition des écrits de Jeanne d'Albret ${ }^{30}$. Outre ses articles sur les textes épistolaires des protestantes ${ }^{31}$, Jane Couchman a signé en 2012 avec Colette H. Winn et François Rouget un ouvrage sur Eléonore de Roye comportant un volet éditorial ${ }^{32}$.

Comme l'illustre cette publication récente, les chercheurs canadiens n’ont pas hésité à collaborer avec des collègues tant américains que français. Ainsi François Rouget a œuvré avec Colette H. Winn pour l'édition des écrits de Louise Boursier ainsi que pour celle des albums de vers de Catherine de

28. Claude La Charité, L'Instruction pour les jeunes dames (1572) de Marie de Romieu : un traité de savoir-" paraître » à l'usage des femmes (thèse de doctorat, Paris : Université Paris IV-Sorbonne, 2000).

29. Diane Desrosiers, William Kemp, Isabelle Crevier et Elisabeth Wengler, éd., Marie d'Ennetières, Euvres (Genève : Droz, à paraître).

30. Eugénie Pascal, Liens de famille, pratiques de pouvoir, conscience de soi. Princesses épistolières au tournant du XVII siècle (Paris : Université de Paris III-Sorbonne nouvelle, 2004) ; Eugénie Pascal, éd., Jeanne d'Albret, Lettres de la Royne de Navarre, avec une ample Declaration d'icelles (Paris : Champion, à paraître).

31. Jane Couchman, “'Our Little Darlings': Huguenot Children and Child-Rearing in the Letters of Louise de Coligny”, in Gender and Early Modern Constructions of Childhood, éd. Naomi J. Miller et Naomi Yavneh (Farnham : Ashgate, 2011), 101-116; "Resisting Henry IV, Catherine de Bourbon and Her Brother", in Sibling Relations and Gender in the Early Modern World: Sisters, Brothers, and Others, éd. Naomi J. Miller et Naomi Yavneh (Aldershot : Ashgate, 2006), 64-76; « Lettres de Louise de Coligny, princesse d'Orange, aux membres de sa famille aux Pays-Bas et en France (1573-1620) », in Lettres de femmes: textes inédits et oubliés du XVI ${ }^{e}$ au XVII siècles, éd. Elizabeth Goldsmith et Colette Winn (Paris : Champion, 2005), 89-134 ; “Charlotte de Bourbon's Correspondence: Using Words to Implement Emancipation", in Women Writers in Pre-Revolutionary France : Strategies of Emancipation, éd. Colette H. Winn et Donna Kuizenga (New York : Garland, 1997), 101-115.

32. Jane Couchman et Colette H. Winn avec la collaboration de François Rouget, éd., Autour d'Eléonore de Roye, princesse de Condé : étude du milieu protestant dans les années 1550-1565 à partir de documents authentiques nouvellement édités (Paris, Genève : Champion, Slatkine, 2012). 
Clermont et de Marguerite de Valois ${ }^{33}$. D'autres réalisations, cette fois dans le domaine de l'analyse littéraire, témoignent de ces efforts soutenus de concertation. Ainsi convient-il de souligner le rôle de Jane Couchman dans le tout récent Ashgate Research Companion to Women and Gender in Early Modern Europe $e^{34}$ et de mentionner la part active que les chercheurs canadiens ont prise dans la constitution du Dictionnaire des femmes de l'Ancienne France (SIEFAR), du Dictionnaire universel des créatrices et de l'Encyclopedia of Women in the Renaissance ${ }^{35}$. Il est intéressant, par ailleurs, de constater le nombre significatif de Canadiens qui ont contribué aux deux ouvrages collectifs dirigés par Colette H. Winn dans la collection "Approaches to Teaching " de la Modern Language Association of America : le premier consacré aux questions pédagogiques relatives à l'Heptaméron de Marguerite de Navarre ${ }^{36}$ et le second à l'enseignement des textes féminins de la Renaissance ${ }^{37}$. L'idée même de s'interroger sur les modalités didactiques de ce corpus aurait été

33. François Rouget et Colette H. Winn, éd., Louise Boursier, Le Récit véritable de la naissance de Messeigneurs et Dames les enfants de France et Instruction à ma fille de Louise Boursier (Genève : Droz, 2000) ; François Rouget et Colette H. Winn, éd., L'Album de vers de Catherine de Clermont, maréchale de Retz (Paris : Champion, 2004) ; François Rouget et Colette H. Winn, éd., L'Album de vers manuscrit de Marguerite de Valois (manuscrit 816 de la Bibliothèque de la Société de l'Histoire du Protestantisme Français) (Paris : Classiques Garnier, 2009). François Rouget a également dirigé avec cette fidèle collaboratrice un numéro spécial de la revue Renaissance et Réforme / Renaissance and Reformation 28.1 (2006) portant sur sur les salons littéraires de femmes à la Renaissance.

34. Allyson M. Poska, Jane Couchman, Katherine A. McIver, éd., The Ashgate Research Companion to Women and Gender in Early Modern Europe (Farnham : Ashgate, 2013). Jane Couchman a aussi collaboré à la Feminist Encyclopedia of French Literature (Westport : Greenwood Press, 1999).

35. Le Dictionnaire des femmes de l'Ancienne France est disponible en ligne à l'adresse électronique suivante : http://www.siefar.org/dictionnaire/fr/Accueil Voir. Béatrice Didier, Antoinette Fouque et Mireille Calle-Gruber, éd., Le Dictionnaire universel des créatrices (Paris : Éditions des femmes, 2013, 3 vol.) et Diana Robin, Anne R. Larsen et Carole Levin, éd., Encyclopedia of Women in the Renaissance. Italy, France, and England (Santa Barbara : ABC-Clio, 2007). Mentionnons également L'histoire des créatrices : l’Antiquité, le Moyen âge, la Renaissance de Liliane Blanc (Montréal : Éditions Sisyphe, 2008).

36. Nancy M. Frelick, "Reading Violent Truths" et Jane Couchman, "Teaching the Heptameron in Marguerite de Navarre's Letters", in Approaches to Teaching Marguerite de Navarre's Heptameron, éd. Colette H. Winn (New York : Modern Language Association of America, 2007), 113-117 et 122-127.

37. Jean-Philippe Beaulieu, "Feminine Authorial Ethos: The Use of Discours sur ce livre as an Introduction to Her Collected Works"; Jane Couchman , "Gender and Genres: Teaching Women and the Epistolary Genre" ; Nancy M. Frelick, "Reading Hélisenne with Transference" ; Claude La Charité, "How Should Sixteenth-Century Feminine Poetry be Taught? The Exemple Case of Marie de Romieu”, etc., in Teaching 
inimaginable il y a à peine vingt ans ; ce changement s'explique en grande partie par la remarquable effervescence critique qui, depuis le début des années 1990, a conféré une légitimité à cette production textuelle et à son étude ${ }^{38}$.

L'apport des chercheurs canadiens à ces développements critiques se singularise par l'angle sous lequel ils abordent l'analyse des textes. Se situant au confluent des traditions épistémologiques française et anglo-saxonne, leur approche se caractérise par la prise en compte des impératifs de l'érudition, mais également par l'attention qu'ils accordent aux modalités d'énonciation de la parole féminine mises en évidence par les gender studies. Ils formulent ainsi des propositions de lecture nourries des courants critiques récents (féminisme, psychanalyse, déconstructionnisme), mais qui tiennent aussi compte de l'historicité des textes ${ }^{39}$. De la sorte, ils échappent généralement à la tentation apologétique qui consisterait à se servir des femmes écrivains d'Ancien Régime comme caution d'a priori idéologiques modernes, telles les revendications d'accès à l'égalité qui, parce qu'étrangères à leur époque, risquent d'occulter l'altérité singulière du passé.

C'est notamment par le biais de la rhétorique que s'actualisent ces avancées critiques et que se concrétise cet intérêt pour la généricité, c'est-à-dire la dimension des textes ressortissant à l'identité sexuelle de leur auteur. Ainsi, à l'occasion du congrès de la Société internationale d'histoire de la rhétorique, à Saskatoon en 1997, Christine Mason Sutherland, qui est professeure émérite à l’Université de Calgary, a organisé une séance sur l'éloquence au féminin dont

French Women Writers of the Renaissance and Reformation, éd. Colette H. Winn (New York : Modern Language Association of America, 2011), 170-180 ; 143-152 ; 206-217 ; 230-241.

38. En témoigne aussi l'importante entreprise éditoriale poursuivie par le Centre for Reformation and Renaissance Studies, Iter et les Presses de l'Université de Toronto avec la série "Other Voice in Early Modern Europe". On trouve notamment parmi les titres de cette collection deux éditions bilingues : Jeanne Flore, Tales and Trials of Love. A Bilingual Edition and Study, éd. et trad. Kelly Digby Peebles et Marta Rijn Finch (Toronto : Centre for Reformation and Renaissance Studies, 2014) et Pernette du Guillet, Complete Poems. A Bilingual Edition, éd. Karen Simroth James et trad. Marta Rijn Finch (Toronto : Centre for Reformation and Renaissance Studies, 2010).

39. À titre d'exemple, signalons les travaux de Nancy M. Frelick qui proposent des interprétations faisant appel entre autres aux outils de la critique postmoderne : "Female Infidelity, Ideology, Subversion and Feminist Practice in Marguerite de Navarre's Heptameron”, Dalhousie French Studies 56 (2001) : 17-26; "Fetishism and Storytelling in Nouvelle 57 of Marguerite de Navarre's Heptameron", in Distant Voices Still Heard. Contemporary Readings of French Renaissance Literature, éd. John O’Brien et Malcolm Quainton (Liverpool : Liverpool University Press, 2000), 138-154. 
les actes ont été rassemblés sous le titre The Changing Tradition. Women in the History of Rhetoric ${ }^{40}$. En outre, le premier colloque spécifiquement consacré à la rhétorique au féminin s'est tenu à l'Université Queens et a donné lieu à la publication du premier ouvrage en français sur le sujet ${ }^{41}$. Dans une perspective plus strictement historique, les actes du colloque de Rimouski (2005), sous la direction de Claude La Charité et de Roxanne Roy, réunissaient de nombreuses contributions de Canadiens ${ }^{42}$. Ces divers travaux ont attiré l'attention sur les liens complexes qu'entretiennent les femmes avec la tradition et les pratiques rhétoriques, en grande partie grâce à la notion d'ethos. En effet, celle-ci s'est révélée centrale pour notre compréhension du déploiement de la rhétorique au féminin, puisqu'elle est directement liée à l'émergence et à l'affirmation d'un sujet traditionnellement tenu à l'écart de la sphère publique. Objet de vives controverses à la Renaissance ${ }^{43}$, le statut de la nature féminine imposait en effet

40. Christine Mason Sutherland et Rebecca Sutcliffe, éd., The Changing Tradition. Women in the History of Rhetoric (Calgary : University of Calgary Press, 1999). Christine Mason Sutherland signe également le chapitre intitulé "Medieval and Renaissance Rhetorical Studies of Women", in The Sage Handbook of Rhetorical Studies, éd. Andrea A. Lunsford, Kirt H. Wilson et Rosa A. Eberly (Los Angeles : Sage Publications, 2009), 53-65.

41. Annette Hayward, éd., La Rhétorique au féminin (Québec : Éditions Nota bene, 2006). De façon plus spécifique, on se reportera au texte de Diane Desrosiers, «Les femmes et la rhétorique au XVI siècle français ", 83-101.

42. Claude La Charité et Roxanne Roy, éd., Femmes, rhétorique et éloquence. Parmi les quatorze contributions canadiennes, notons le bilan synthétique que propose Diane Desrosiers, "Femmes et rhétorique. État présent de la recherche », 13-21.

43. Marc Angenot a joué un rôle précurseur en matière d'analyse du discours social; dans son ouvrage devenu un classique : Les champions des femmes : examen du discours sur la supériorité des femmes, 1400-1800 (Montréal : Presses de l’Université du Québec, 1977), il répertoriait l'ensemble des lieux communs constitutifs de la « nature » féminine. Marie Claude Malenfant a donné suite à cette entreprise en commentant les argumentaires pro et contra relatifs à la femme, dans le livre tiré de sa thèse de doctorat: Argumentaires de l'une et l'autre espèce de femme : le statut de l'exemplum dans les discours littéraires sur la femme (1500-1550) (Québec : Presses de l'Université Laval, 2003). De son côté, RenéeClaude Breitenstein, professeure à l'Université Brock, a montré comment s'élabore progressivement un discours de célébration du sexe féminin qui en redéfinit l'objet (La rhétorique encomiastique dans les éloges collectifs de femmes imprimés de la première Renaissance française (1493-1555) [thèse de doctorat, Montréal : Université McGill, 2008]). Un numéro de la revue Études françaises est consacré à cette problématique (Renée-Claude Breitenstein, éd., Publics et publications dans les éloges collectifs de femmes à la fin du Moyen Âge et sous l'Ancien Régime 47.3 [2011]). Sous un éclairage différent, Hélène Hotton rend compte de l'altérité du féminin dans les discours démonologiques (Les marques du diable 
le choix de certaines modalités énonciatives afin de légitimer les prises de parole des femmes de plus en plus nombreuses et variées. Il n'est donc pas étonnant que la question de l'ethos au féminin apparaisse comme une avenue de recherche féconde. En font foi deux collectifs, dirigés par des collègues québécois, qui explorent les stratégies de mise en scène de soi chez les femmes écrivains ${ }^{44}$. Ces divers travaux ont notamment montré que l'expression féminine passe par des formes discursives plus rattachées au sermo qu'à l'oratio, c'est-à-dire à une prise de parole au « je » qui n'est pas subordonnée aux règles de la contentio et dont relèvent, par exemple, l'art épistolaire et la conversation. Dès 1994, l'épistolarité au féminin a suscité l'intérêt des chercheurs ${ }^{45}$. Jane Couchman, Luc Vaillancourt et Eugénie Pascal ont ainsi étudié la correspondance féminine à la lumière de ses modalités matérielles et thématiques ${ }^{46}$. Ils ont mis en évidence des topiques

et les signes de l'Autre : rhétorique du dire démonologique à la fin de la Renaissance (thèse de doctorat, Montréal : Université de Montréal, 2011) ; L’Autre féminin dans les traités de démonologie (1550-1620) [mémoire de maîtrise, Montréal : Université McGill, 2002]). Ce sont aussi ces représentations de la femme sous les traits de la sorcière qui ont intéressé Patrick Snyder, professeur à l'Université de Sherbrooke (Trois figures du diable à la Renaissance : l'enfant, la femme et le prêtre [Montréal : Fides, 2007] ; Représentations de la femme et chasse aux sorcières. XIII $-X V^{e}$ siècles [Montréal : Fides, 2000]).

44. Jean-Philippe Beaulieu et Andrea Oberhuber, éd., Jeu de masques. Les femmes et le travestissement textuel 1500-1940 (Saint-Étienne : Publications de l'Université de Saint-Étienne, 2011) ; Claude La Charité, éd., Masques et figures du sujet féminin aux XVI et XVII siècles, Tangence 77 (2005). JeanPhilippe Beaulieu signe également plusieurs articles où il aborde les enjeux identitaires liés à la ventriloquie féminine ; voir notamment « Les voix de la maréchale d'Ancre. Effets de ventriloquie dans quelques pamphlets de 1617 ", in La pseudonymie d'auteur dans la littérature française (XVI ${ }^{e}-X X I^{e}$ siècles), éd. David Martens et Myriam Watthee-Delmotte (Louvain-La-Neuve : Presses Universitaires de Louvain, à paraître) ; "Lettre de femme, voix d'homme? Jeux identitaires et effets de travestissement dans la treizième épître familière d'Hélisenne de Crenne ", in Postures et impostures de l'individualisme humaniste, éd. Luc Vaillancourt, Tangence 84 (2007) : 31-47 ; "Cette villageoise est Profetisse". Jeanne d'Arc et la figure politique de la sibylle au XVII siècle ", in La Passion des lettres. Études de littérature médiévale et québécoise en hommage à Yvan Lepage, éd. Pierre Berthiaume et Christian Vandendorpe (Ottawa : Éditions David, 2006), 141-152.

45. À dix ans d'intervalle, deux colloques internationaux se tenaient au Canada sur ce sujet : Benoît Melançon et Pierre Popovic, éd., Les femmes de lettres : écriture féminine ou spécificité générique ? (Montréal : Centre universitaire de lecture sociopoétique et des correspondances, Université de Montréal, 1994) et Claude La Charité, éd., La Rhétorique épistolaire sous l’Ancien Régime : de la théorie aux pratiques (Québec: Presses de l'Université Laval, à paraître).

46. Jane Couchman et Ann Crabb, éd., Women's Letters Across Europe, 1400-1700: Form and Persuasion (Aldershot : Ashgate, 2005); Luc Vaillancourt, La lettre familière au XVI siècle. Rhétorique humaniste de l'épistolaire (Paris : Champion, 2003) ; Eugénie Pascal, Liens de famille, pratiques de pouvoir, conscience 
qui s'articulent autour des relations familiales et des moments significatifs de l'expérience des femmes (naissances ${ }^{47}$, unions conjugales, maladies, etc.). L'importance de la sphère domestique, qui détermine en quelque sorte la matière et la manière de leurs échanges épistolaires, a incité divers chercheurs à se pencher sur ses manifestations aux différents âges de la vie : l'enfance ${ }^{48}$ et la vieillesse ${ }^{49}$, ou dans certaines institutions, comme le célibat ou le mariage ${ }^{50}$.

Si la pratique de formes et de types de discours moins valorisés à l'époque est l'un des traits que les études récentes ont contribué à préciser, l'exercice littéraire que représente la traduction a en revanche permis aux femmes de s'inscrire au cour même des pratiques privilégiées par l'humanisme. Elles ont ainsi participé à l'effort de diffusion des connaissances en langue vernaculaire au début de l'époque moderne. Toutefois, à l'instar d'autres activités scripturaires associées aux femmes, tels les échanges épistolaires,

de soi. Princesses épistolières au tournant du XVII siècle (Paris : Université de Paris III-Sorbonne nouvelle, 2004). Les épistolières des XVII e ${ }^{e}$ XVIII ${ }^{e}$ siècles français ont aussi beaucoup retenu l'attention : voir Marie-France Silver et Marie-Laure Girou Swidersky, éd., Femmes en toutes lettres : les épistolières du XVII siècle (Oxford : Voltaire Foundation, 2000) et les nombreux articles sur Madame de Sévigné que signe Constance Cartmill, professeure à l'Université du Manitoba.

47. Voir Brenda Dunn-Lardeau, "Prodigious Births and Death in Childbirth in Le Palais des nobles Dames (Lyon, 1543)," Renaissance et Réforme / Renaissance and Reformation 21.3 (1997) : 43-62. Signalons que Brenda Dunn-Lardeau, professeure à l'Université du Québec à Montréal, a aussi procuré l'édition critique du Palais des nobles Dames de Jean Du Pré (Paris : Champion, 2007).

48. Hélène Cazes, éd., Histoires d'enfants : représentations et discours de l'enfance sous l'Ancien Régime (Québec : Presses de l’Université Laval, 2008).

49. Brenda Dunn-Lardeau, «La vieille femme chez Marguerite de Navarre », Bibliothèque d'humanisme et Renaissance 61.2 (1999) : 375-398.

50. On consultera le site http://mariage.uvic.ca ainsi que le numéro de la revue Dalhousie French Studies 56 (2001) que Claire Carlin, professeure à l'Université Victoria, a consacré au mariage sous l'Ancien Régime. Voir aussi de la même auteure, "Une curiosité vaine et indiscrète" : le mariage au carrefour de la vie privée et de l'intérêt public", in La médiatisation de la vie privée, $X V^{e}-X X^{e}$ siècles, éd. Agnès Walch (Arras : Artois Presses Université, 2012), 152-162 ; "Perfect Harmony. Love and Marriage in Early Modern Pedagogy", in The Art of Instruction: Essays on Pedagogy and Literature in $17^{\text {th }}$-Century France, éd. Anne L. Birberick (Amsterdam : Rodopi, 2008), 201-224. Elle a également dirigé le collectif Imagining Contagion in Early Modern Europe (Basingstoke : Palgrave Macmillan, 2005). Quant au célibat, voir l'article de Brenda Dunn-Lardeau, « La place de La Comedie des quatre femmes de Marguerite de Navarre (1542) dans le discours sur le célibat volontaire comme modèle de félicité de l'Arioste à Gabrielle Suchon », Renaissance et Réforme / Renaissance and Reformation 26.4 (2002) : 113-139. 
la traduction renvoie à une posture tributaire des formes de la modestie rhétorique - mode idéal de légitimation de la voix féminine - c'est-à-dire à une façon pour les femmes de se positionner en coulisses de la notoriété. En effet, à une époque où la traduction apparaît comme l'un des vecteurs du savoir, elle fait participer les femmes à ces transferts culturels, de l'Antiquité vers le monde contemporain, mais d'une façon oblique, indirecte. Par-delà ses vertus pédagogiques, la traduction a ainsi permis à plusieurs femmes de la Renaissance de s'assurer une place dans le monde des lettres en se faisant le truchement d'auteurs majeurs. Le collectif D'une écriture à l'autre : les femmes et la traduction sous l'Ancien Régime ${ }^{51}$ a bien montré la diversité des fonctions dévolues à l'activité traductrice des femmes, qui constitue encore un objet d'étude prometteur comme l'illustre un numéro récent de la revue Renaissance et Réforme / Renaissance and Reformation ${ }^{52}$.

Par contraste avec une situation qui a longtemps prévalu, si, à titre d'exemple, on reconnaît désormais que les écrits d'Hélisenne de Crenne (qui forment le plus large corpus féminin imprimé au cours de la première moitié $d u$ $\mathrm{XVI}^{\mathrm{e}}$ siècle, devant celui de Marguerite de Navarre) justifient que l'on interroge plus en profondeur leur mise en œuvre, cela n'est pas étranger, entre autres, à la somme imposante de contributions canadiennes qui lui ont été consacrées jusqu'à maintenant. Rappelons que la première journée d'études portant exclusivement sur Hélisenne de Crenne s'est tenue à Montréal et qu'elle a donné lieu non seulement à un collectif ${ }^{53}$, mais aussi à toute une série d'éditions et d'analyses publiées par la suite. Ce renouveau d'intérêt est aussi vrai pour d'autres figures féminines qui, grâce notamment aux études menées par des chercheurs canadiens, ont émergé de l'ombre dans laquelle elles avaient été reléguées au cours des derniers siècles. Sous la plume des commentateurs et des traducteurs, bon nombre de femmes de lettres ont ainsi pris un nouveau relief :

51. Jean-Philippe Beaulieu, éd., D’une écriture à l'autre. Les femmes et la traduction sous l'Ancien Régime (Ottawa : Les Presses de l'Université d'Ottawa, 2004).

52. Marie-Alice Belle, éd., La traduction au féminin en France et en Angleterre (XVI et XVII siècles) / Women's Translations in Early Modern England and France, Renaissance et Réforme / Renaissance and Reformation 35.4 (2012).

53. Jean-Philippe Beaulieu et Diane Desrosiers-Bonin, éd., Hélisenne de Crenne. L'écriture et ses doubles (Paris : Champion, 2004). 
Anne de Graville ${ }^{54}$, Marie de Romieu ${ }^{55}$, Anne de Marquets ${ }^{56}$. De Christine de Pizan ${ }^{57}$ jusqu’à Marie de Gournay ${ }^{58}$, les travaux réalisés par les chercheurs

54. Voir les travaux de Mawy Bouchard, professeure à l’Université d'Ottawa : "La translation du “vieil langaige et prose, en nouveau et rime" : Anne de Graville et les visées épidictiques du Beau romant ", in La traduction au féminin en France et en Angleterre (XVI et XVII siècles) / Women's Translations in Early Modern England and France, éd. Marie-Alice Belle, Renaissance et Réforme / Renaissance and Reformation 35.4 (2012) : 5-23 ; «Les belles (in)fidèles ou la traduction de l'ambiguïté masculine : les Rondeaux d'Anne de Graville », Neophilologus 88.2 (2004) : 189-202 ; "Anne de Graville (1492-1544) et la tradition épique au XVI e siècle ", in L'Écriture des femmes à la Renaissance française, éd. Diane Desrosiers-Bonin, Littératures 18 (1998) : 31-63 ; «Le roman “épique” : l'exemple d'Anne de Graville », Études françaises 32.1 (1996) : 99-107.

55. Claude La Charité, « Marie de Romieu et l'écriture androgyne : les marques de généricité du sujet lyrique dans Les Premieres ceuvres poetiques (1581) », Sextant 17/18 (2002) : 213-234; "L'aporie de l'épître dédicatoire à la Renaissance : le cas de Marie de Romieu ", Revue canadienne d'études rhétoriques / Canadian Journal of Rhetorical Studies 13 (2002) : 15-28; «Du Dialogo de la bella creanza de le donne (1539) d'Alessandro Piccolomini à l'Instruction pour les jeunes dames (1572) de Marie de Romieu ou quand le paradoxe fait l'opinion ", Réforme Humanisme Renaissance 53 (2001) : 103-112 ; " "Ce male vers enfant de ta verve femelle" : les destinataires féminins de la lyrique amoureuse de Marie de Romieu ", Nouvelle Revue du XVI siècle 18.2 (2000) : 81-94 ; "Le problème de l'attribution de l'Instruction pour les jeunes dames (1572) et l'énigmatique cryptonyme M. D. R. ", Bibliothèque d'Humanisme et Renaissance 62.1 (2000) : 119-128 ; «Le Dialogo de la bella creanza de le donne (1539) d'Alessandro Piccolomini et ses adaptateurs français ", Renaissance et Réforme / Renaissance and Reformation 23.1 (1999) : 43-57.

56. Après le livre de sœur Mary H. Seiler, Anne de Marquets, poétesse religieuse du XVI siècle (Washington : Catholic University of America Press) publié en 1931, on doit à Hannah Fournier le premier article consacré à Anne de Marquets en laquelle on a longtemps voulu voir un simple épigone des poètes de la Pléiade : "La voix textuelle des Sonets spirituels d'Anne de Marquets ", Études littéraires 20.2 (1987) : 77-92.

57. Claire Le Brun, "Christine de Pizan et la lyrique du deuil », @nalyses 12.3 (2007) ; de la même auteure, "Christine de Pizan et l'édification de la Cité éternelle », Études françaises 37.1 (2001) : 51-65; voir aussi Madeleine Jeay, «Traversée par le verbe : l'écriture de soi comme geste prophétique chez Christine de Pizan ", Dalhousie French Studies 47 (1999) : 7-27, et Eva Kushner, " Trois locutrices du XVI ${ }^{e}$ siècle ; deux miroirs », Dalhousie French Studies 52 (2000) : 14-21.

58. Outre les nombreux articles et éditions que l'on doit à Jean-Philippe Beaulieu et à Constant Venoesen, signalons que Richard Hillman et Colette Quesnel ont traduit en anglais divers écrits de Marie Le Jars de Gournay (Apology for the Woman Writing and Other Works [Chicago : Chicago University Press, 2002]). Richard Hillman a par ailleurs signé plusieurs articles relatifs à la fille d'alliance de Montaigne : «Des Champsfaëz de Claude de Taillemont au labyrinthe du Songe shakespearien, en passant par le Proumenoir de Monsieur de Montaigne », Studi francesi 48.1 (2004) : 3-18; "Les intellectuelles et l'amour : Marie de Gournay et Marguerite de Valois ", Renaissance et Réforme / Renaissance and Reformation 24.4 (2000): 
canadiens auront donc permis depuis deux décennies de mettre en lumière l'expression de la subjectivité des femmes de la Renaissance française ainsi que les modalités de leur façonnement auctorial à l'aube de la première modernité.

115-128, et " Hamlet et la préface de Marie de Gournay », Renaissance et Réforme / Renaissance and Reformation 18.3 (1994) : 29-42. 